\title{
PERIODICITY AND ROOTS OF TRANSFINITE STRINGS
}

\author{
Olivier Carton ${ }^{1}$ and Christian Choffrut ${ }^{2}$
}

\begin{abstract}
This contribution extends the notions of roots and periodicity to strings of transfinite lengths. It shows that given a transfinite string, either it possesses a unique root or the set of its roots are equivalent in a strong way.
\end{abstract}

Mathematics Subject Classification. 68R15.

\section{INTRODUCTION}

The related notions of periodicity, conjugacy and primitivity of strings are without any doubt among the most important since they represent the simplest "regularities" that can be observed. They can be defined in different equivalent ways. We can view a string as mapping an arbitrary integer interval into a finite set of symbols and extend thus the classical definition of analytic periodic real functions, provided one changes the domain from the reals to the integers, [6]. A more "word combinatorical" viewpoint relates periodicity with self-overlapping of strings, i.e., with the "conjugacy" relation. As such, it has been studied in numerous contexts. E.g. Knuth-Morris-Pratt's string-matching algorithm is mainly based on periodicity implemented as the so-called "failure function". Duval studied the set of periodicities of strings, references [4] and [5]. More technically, when trying to "solve" equations in the free monoid, i.e., to find some type of information on the words that satisfy a given equation, the main tool is again the conjugacy relation.

It is not always clear how to generalize the notion of periodicity to other structures. As a "geometric" property (invariance under some type of translation of a given pattern) there are a few tempting candidates. E.g., infinite strings can be investigated from this perspective. Césari initiated the study of local versus

Keywords and phrases: Ordinals, combinatorics on words.

1 Institut Gaspard Monge, CNRS, Université de Marne-la-Vallée 5, boulevard Descartes, 77454 Marne-la-Vallée Cedex 2, France; e-mail: Olivier.Carton@univ-mlv.fr

2 LIAFA, Université Paris 7, 6 étage, bureau 6A7, 175 rue du Chevaleret, 75013 Paris, France; e-mail: Christian.Choffrut@liafa.jussieu.fr 
global periodicity in [2]. Also, it can be spoken in a relevant way of periodicity in trees [7] for example. Another direction of research can be found in [1] where so called semiperiodic words are defined.

In the present work, we focus our attention on strings of transfinite length. The "invariance under translation" cannot be adapted directly as we shall see, because of the peculiarities of the operation of addition of ordinals, so we have to resort to a property of the division. Next to periodicity, we investigate the notion of roots of a transfinite string which can be viewed as specific periods of the string. The purpose of this paper is therefore twofold. It proposes a notion of periodicity of transfinite strings which generalizes that of finite strings and it shows that every transfinite string possesses a unique, properly defined, "primitive root".

\section{Preliminaries}

We refer the reader to the numerous standard handbooks such as $[9,10]$ or $[8]$ for a comprehensive exposition of the theory on ordinals. In particular we assume the reader is familiar with the basic arithmetical rules for ordinals. We consider countable ordinals only and we denote this collection by Ord.

\subsection{Ordinals}

Every ordinal $\alpha$ admits a unique representation, known as Cantor's normal form, as a finite sum of non-increasing prime components. By grouping the equal prime components, an arbitrary non-zero ordinal can thus be written as

$$
\alpha=\omega^{\lambda_{n}} a_{n}+\omega^{\lambda_{n-1}} a_{n-1}+\ldots+\omega^{\lambda_{1}} a_{1}+\omega^{\lambda_{0}} a_{0}
$$

where $n \geq 0, a_{n}, \ldots, a_{1}, a_{0}<\omega, a_{n}, a_{0} \neq 0$ and $\lambda_{n}>\lambda_{n-1}>\ldots>\lambda_{0}$. The ordinal $\lambda_{n}$ is the degree and $\lambda_{0}$ the order of $\alpha$. We recall that $\alpha$ is a successor ordinal if $\lambda_{0}=0$ else it is a limit ordinal. An ordinal of the form $\omega^{\lambda}$ for some ordinal $\lambda>0$ is called a prime component. Prime components are characterized by the condition that they cannot be expressed as the sum of two smaller ordinals.

For the sake of self-containment we recall the basical results on arithmetics of ordinals. Addition of two ordinals is associative and satisfies for all $a, b<\omega$ and all $\mu, \nu \in$ Ord

$$
\omega^{\mu} a+\omega^{\nu} b= \begin{cases}\omega^{\nu} b & \text { if } \nu>\mu \\ \omega^{\mu}(a+b) & \text { if } \nu=\mu\end{cases}
$$

For $\nu<\mu$, the expression cannot be simplified further. Addition is not commutative but characterizing the pairs of ordinals that commute is easy ([10], Th. 1 , p. 346). 
The product of ordinals may also be defined. It is associative, distributes over the sum to the right $(\alpha(\beta+\gamma)=\alpha \beta+\alpha \gamma)$ and satisfies in particular

$\left(\omega^{\lambda_{n}} a_{n}+\omega^{\lambda_{n-1}} a_{n-1}+\ldots+\omega^{\lambda_{0}} a_{0}\right) \alpha= \begin{cases}\omega^{\lambda_{n}+\mu} & \text { if } \alpha=\omega^{\mu}, \text { for } \\ \omega^{\lambda_{n}} a_{n} \alpha+\omega^{\lambda_{n-1}} a_{n-1}+ & \text { some } \mu>0 \\ \ldots+\omega^{\lambda_{0}} a_{0} & \text { if } \alpha<\omega .\end{cases}$

Observe that the product does not distribute to the left, e.g. $(\omega+1) \cdot \omega=\omega^{2} \neq \omega^{2}$ $+\omega$.

It is well-known that every ordinal may be factored, though not necessarily uniquely, as a product of prime ordinals, i.e., ordinals whose unique right divisors are themselves and 1 ([10], Th. 1, p. 309). Furthermore, the prime factors which are successor ordinals are exactly the prime integers and those ordinals of the form $\omega^{\lambda}+1$ where $\lambda$ is an arbitrary non-zero ordinal ([10], Th. 3, p. 336). In other words, infinite prime ordinals which are successor ordinals are exactly the successors of the prime components.

\subsection{TRANSFINITE STRINGS}

Given a finite alphabet $\Sigma$, a string is a mapping $u$ of Ord into $\Sigma$. Equivalently, $u$ is a sequence of $\Sigma$ indexed by an ordinal $\alpha$. We denote by $u_{i}$, for all $i<\alpha$, the image of $i$ in this mapping. The collection of all strings is denoted by $\Sigma$ Ord. The ordinal $\alpha$ is the length of $u$, denoted by $|u|$. The empty string, denoted by 1 , is the string of length 0 and is the unit of $\Sigma$ Ord as a monoid. By extension, the degree of a string $x$ is the degree of its length. For $a \in \Sigma,|u|_{a}$ denotes the length in the letter $a$ of the string $u$, i.e., the ordinal of the subsequence consisting of all the positions $i<\alpha$ for which $u_{i}=a$.

The concatenate of two strings $u$ and $v$ is the mapping from the ordinal $|u|+|v|$ to $\Sigma$ defined by

$$
(u v)_{i}= \begin{cases}u_{i} & \text { if } i<|u| \\ v_{j} & \text { if } i=|u|+j, j<|v| .\end{cases}
$$

Observe that $|u v|=|u|+|v|$ holds for all $u, v \in \Sigma^{\text {Ord }}$ by definition, but the condition $u v=w,|u|>0$ does not imply $|v|<|w|$ (consider $u=a, v=w=a^{\omega}$ ), which is a main departure from finite strings. Another difference is the fact that concatenation (also called product), like addition for ordinals, is left cancellative but not right cancellative $\left(a^{\omega} a a^{\omega}=a^{\omega} a^{\omega}\right)$. Also, concatenation can be defined for a collection of strings indexed by an arbitrary ordinal. As a particularly important 
case, given a string $u$, we denote by $u^{\omega}$ the string infinitely concatenated with itself, i.e., the string of length $|u| \omega$ defined by

$$
u_{\ell k+i}^{\omega}=u_{i}
$$

where $\ell=|u|, k<\omega$ and $i<\ell$.

The notion of prefix extends naturally from finite to arbitrary strings. An occurrence of a string $w \in \Sigma^{\text {Ord }}$ in a string $u \in \Sigma^{\text {Ord }}$ is a pair $(\alpha, w) \in$ Ord $\times \Sigma^{\text {Ord }}$ such that $u=x w y$ for some strings $x, y \in \Sigma^{\text {Ord }}$ with $|x|=\alpha$. For example, consider the string $x=y y$ where $y=a b a^{2} b a^{3} b \ldots a^{i} b \ldots$ of length $\omega$. The underlined occurrence $a b a^{2} b \underline{a^{3} b} \ldots a^{i} b \ldots a b a^{2} b \ldots a^{i} b \ldots$ is specified by the pair $\left(5, a^{3} b\right)$ while the underlined occurrence $a b a^{2} b a^{3} b \ldots a^{i} b \ldots a b \underline{a^{2} b} \ldots a^{i} b \ldots$ is specified by the pair $\left(\omega+2, a^{2} b\right)$. The occurrences of a given string are linearly ordered by their first component, i.e. $\left(\alpha_{1}, w_{1}\right)$ is before $\left(\alpha_{2}, w_{2}\right)$ if $\alpha_{1}<\alpha_{2}$.

We may also say that two strings $x, y \in \Sigma^{\text {Ord }}$ are comparable if $x$ is a prefix of $y$ or $y$ is a prefix of $x$. The most elementary property of finite strings is known as Levi's lemma. It trivially holds for strings of arbitrary lengths. We recall it here.

Proposition 2.1. Let $x, y, z, t \in \Sigma^{\text {Ord }}$ satisfy the equality $x y=z t$. Then there exists $u \in \Sigma$ Ord such that either $x=z u$ and $t=u y$ or $z=x u$ and $y=u$ tholds.

\section{PERIODICITY}

Mimicking the definition for functions, the period of a finite or infinite string $u$ is defined as an integer $p$ less than the length $|u|$, such that for all $0 \leq i<i+p<|u|$, $u_{i}=u_{i+p}$ holds. The addition of ordinals is not commutative, so if we are to extend this notion in a naive way, we must choose between equality $u_{i}=u_{i+p}$ or equality $u_{i}=u_{p+i}$. The first equality implies however that $u_{i}$ is constant for all ordinals $i$ of degree less than or equal to that of $p, e . g$., the string $u=a^{\omega} a v$ where $a$ is a letter and $v$ is an arbitrary string of length $\omega$ would have period 1 . The opposite equality carries no information when the degree of $i$ is greater than that of $p$ and with this definition, $(a b)^{\omega} v$ would be periodic of period 2 for an arbitrary $v$. We are thus lead to attack the problem differently, via the operation of division. The uniqueness property of the quotient and remainder for the integers can be extended to all ordinals, provided the quotient is multiplied to the right of the divisor. Otherwise, it may no longer hold $(e . g ., \omega \cdot 3+2=(\omega+1) \cdot 3+1)$.

Proposition 3.1 ([10], Th. 2, p. 298). Let $\alpha, \beta$ be two ordinals. There exists one and only one pair of ordinals $\lambda, \rho$ such that $\rho<\beta$ and $\alpha=\beta \lambda+\rho$.

With this in mind, given two ordinals $\alpha$ and $\beta$, we define the equivalence $\underset{\beta}{\sim}$ on all ordinals less than $\alpha$ by setting $i \underset{\beta}{\sim} j$ whenever the two ordinals $i, j<\alpha$ have the same remainder in the division by $\beta$, i.e., whenever there exist three ordinals $\lambda, \mu$ and $\rho<\beta$ such that $i=\beta \cdot \lambda+\rho, j=\beta \cdot \mu+\rho$ holds.

The first result serves as a definition of the notion of periodicity. 
Proposition 3.2. Given a string $x$ and an ordinal $\beta$, the following conditions are equivalent:

i) there exists a string $y$ of length $\beta$ such that $x$ is a prefix of $y^{\tau}$ for some ordinal $\tau$;

ii) for all ordinals $i, j<|x|$, if $i \underset{\beta}{\sim} j$ holds then we have $x_{i}=x_{j}$.

Proof. Indeed, if i) holds then for all ordinals $i, j<|x|$ having the same remainder $\rho$ in the division by $\beta$ of $y$, we have $x_{i}=x_{j}=y_{\rho}$. Conversely, let $y$ be the prefix of $x$ of length $\beta$ and let $\tau$ be an ordinal such that $|x| \leq \beta \cdot \tau$. For each $0 \leq i<|x|$ consider the division: $i=\beta \cdot \lambda+\rho$. Then we have $x_{i}=y_{\rho}=y_{\beta \cdot \lambda+\rho}^{\tau}$ which completes the proof.

The string $y$ of the previous proposition is called a period of $x$. By extension we say that the length of $y$ is a period of $x$. Whichever is meant will be clear from the context.

\section{Roots}

A period of a string $x$ has been defined as a string $y$ such that $x$ is a prefix of some power of $y$. Here we impose that $x$ be equal to some power of $y$, in which case we say $y$ is a root of $x$. A root is "primitive" when it cannot be decomposed further. For transfinite strings unicity of the primitive root does not necessarily hold. It is only guaranteed when some technical condition is met on the exponents of the string. When this condition is not satisfied, we must restrict the notion of primitivity in order to keep the unicity.

In order to establish this result we recall some notions and results of [3].

\subsection{Generalized CyClicity}

The idea behind the following definition is to express a property on two transfinite strings which guarantees that any two arbitrary countable products of these two strings are comparable.

Definition 4.1. Two strings $x, y \in \Sigma^{\mathrm{O} \text { rd }}$ are generalized cyclic if

i) either there exists a string $u \in \Sigma$ Ord and two ordinals $\alpha, \beta \in$ Ord such that $x=u^{\alpha}, y=u^{\beta}$

ii) or there exist two strings $u, v \in \Sigma^{\mathrm{Ord}}$, two ordinals $\alpha, \beta \geq 0$ and two integers $0 \leq i, j<\omega$ such that

$$
x=\left(u^{\omega} v\right)^{\alpha} u^{i} \quad y=\left(u^{\omega} v\right)^{\beta} u^{j} \quad \text { with } \alpha=0 \text { if and only if } \beta=0 .
$$

The term "generalized cyclic" comes from the fact that whatever the product of occurrences of $x$ and $y$, the result is of the form $u^{\gamma}$ in case i) or in case ii) when $\alpha=\beta=0$ or of the form $\left(u^{\omega} v\right)^{\gamma} u^{r}$ for some $\gamma \in$ Ord and $0 \leq r<\omega$ in the remaining case ii). Actually $r$ may assume the values $0, i$ and $j$ only. 
Example 4.2. The strings $x=\left(a^{\omega} b\right)^{\omega} a^{2}$ and $y=a^{\omega} b a$ are generalized cyclic but $x=\left(a^{\omega} b\right)^{2} a^{2}$ and $y=a$ are not (in particular, $a^{\omega \cdot 2}$ is not a prefix of $\left(a^{\omega} b\right)^{2} a^{2}$, see condition iv) of the next proposition).

Proposition 4.3. Let $x$ and $y$ be two strings. The following conditions are equivalent:

i) the two strings $x$ and $y$ are generalized cyclic;

ii) for all ordinals $\lambda$ and $\mu$, the two strings $x^{\lambda}$ and $y^{\mu}$ are comparable;

iii) for some limit ordinals $\lambda$ and $\mu$, we have $x^{\lambda}=y^{\mu}$;

iv) for some ordinals $\lambda$ and $\mu$ the two strings $x^{\lambda}$ and $y^{\mu}$ have a common prefix of length $\max \{|x y|,|y x|\}$.

\subsection{Strong COnjugacy}

Conjugacy is a property that can be defined on all monoids. Formally, two elements $x, y$ are conjugate if there exist two elements $u, v$ such that $x=u v$ and $y=v u$ holds. The following refines the notion of conjugacy.

Definition 4.4. Two strings $x, y \in \Sigma^{\mathrm{Ord}}$ are strongly conjugate if and only if there exist $z, t \in \Sigma$ Ord and two integers $m, n$ such that

$$
x=z^{\omega} t z^{n} \text { and } y=z^{\omega} t z^{m} .
$$

We also say that $x$ and $y$ are strong conjugates. Observe that if $n \leq m$ which can be assumed without loss of generality, we have: $x=z^{m-n} z^{\omega} t z^{n}$ and $y=z^{\omega} t z^{n} z^{m-n}$, i.e., strong conjugacy implies conjugacy. The converse is not true, e.g., $a^{\omega} b$ and $b a^{\omega}$ are conjugate but not strongly conjugate. Strong conjugacy has nice properties, see [3], in particular that of being an equivalence relation.

\subsection{UNICITY OF THE ROOT}

Definition 4.5. A string $x \in \Sigma^{\mathrm{Ord}}$ is primitive if $x=y^{\alpha}$ implies $\alpha=1$. It is strongly primitive if all its strong conjugates are primitive.

Example 4.6. $\left(a^{\omega} b\right)^{2} a$ is not primitive since it is equal to $\left(a^{\omega} b a\right)^{2},\left(a^{\omega} b\right)^{\omega} a$ is primitive since its length is $\omega^{2}+1$ but not strongly primitive since it is strongly conjugate to $\left(a^{\omega} b\right)^{\omega}$. Finally, $a^{\omega} b a^{2}$ is strongly primitive since its conjugates have length $\omega+n$ for some $n<\omega$.

Definition 4.7. A root of a non-empty string $x \in \Sigma^{\text {Ord }}$ is a string $y$ such that $x=y^{\alpha}$ for some ordinal $\alpha$. Then we say that $\alpha$ is an exponent of $x$ which is associated with $y$.

Observe that the exponent may assume a finite number of values only, since it is a right-hand divisor of $|x|, c f$. [10] (Th. XIV 12. 1). 
The main result concerning the notions of root and exponent for transfinite strings is the following:

Proposition 4.8. If a non-empty string has an exponent which is a successor ordinal, then it has a unique primitive root. It is the shortest among its roots.

If it has an exponent which is a limit ordinal, the set of its strongly primitive roots is an equivalence class of the relation of strong conjugacy.

Observe that discriminating the two cases is necessary since e.g., $a^{\omega} b,\left(a^{\omega} b\right)^{\omega} a$, $\left(a^{\omega} b\right)^{\omega^{2}} a, \ldots,\left(a^{\omega} b\right)^{\omega^{n-1}} a$ are primitive roots of $\left(a^{\omega} b\right)^{\omega^{n}}$ but among these $n$ primitive roots, $a^{\omega} b$ only is strongly primitive.

Proof. Since there exists no infinite descending chain, it is clear that every string admits a primitive root. We start with three general claims.

Claim 1. If $y_{1}$ and $y_{2}$ are two primitive roots of $x$, then there exist $u, v \in \Sigma$ Ord, $0 \leq i_{1}, i_{2}<\omega$ and $\epsilon_{1}, \epsilon_{2} \in$ Ord such that $y_{1}=\left(u^{\omega} v\right)^{\epsilon_{1}} u^{i_{1}}$ and $y_{2}=\left(u^{\omega} v\right)^{\epsilon_{2}} u^{i_{2}}$ hold.

This is a reformulation of Proposition 4.3.

The next claim concerns the exponent $\epsilon$ in the expression $\left(u^{\omega} v\right)^{\epsilon} u^{i}$ when it is assumed to denote a primitive string.

Claim 2. Assume $y=\left(u^{\omega} v\right)^{\epsilon} u^{i}$ is primitive with $u, v \in \Sigma^{\mathrm{Ord}}, 0 \leq i<\omega$ and $\epsilon \in$ Ord. If $\epsilon>1$ then $\epsilon$ is an infinite prime component of the form $\omega^{\tau}$ for some ordinal $\tau>0$ and furthermore we have $i \neq 0$.

Indeed, condition $\epsilon>1$ implies $i \neq 0$ trivially. Consider the special case $y=\left(a^{\omega} b\right)^{\epsilon} a$ where $a, b \in \Sigma$, i.e., $u$ and $v$ are reduced to letters. The ordinal $\epsilon$ is not a successor ordinal since otherwise equality $\left(a^{\omega} b\right)^{\epsilon} a=\left(a^{\omega} b a\right)^{\epsilon}$ would hold, contradicting the primitivity of $y$. If $\epsilon+1$ is not a prime component, then by [10] (Th. 3, p. 336) we can factor $\epsilon+1=\left(\omega^{\gamma}+1\right)\left(\epsilon^{\prime}+1\right)$ where $\epsilon^{\prime}$ is a non-zero limit ordinal. A simple computation shows that this implies $\omega \epsilon+1$ $=\left(\omega^{1+\gamma}+1\right)\left(\epsilon^{\prime}+1\right)$. However, $\omega \epsilon+1$ is the length of $y$ and $\omega^{1+\gamma}+1$ is the length of the prefix $z=\left(a^{\omega} b\right)^{\omega^{\gamma}} a$ of $y$, yielding $y=z^{\epsilon^{\prime}+1}$ contradicting the primitivity of $y$. The general case can be dealt with as follows. Consider the substitution $\varphi(a)=u^{i}$ and $\varphi(b)=v$. Then the string $y$ of the claim equals $\varphi\left(\left(a^{\omega} b\right)^{\epsilon} a\right)$ and $\left(a^{\omega} b\right)^{\epsilon} a$ is primitive else $y$ would not be primitive either. The conclusion follows.

Claim 3. If a non-empty string $x$ has an exponent associated with a primitive root which is a successor ordinal, then all other exponents associated with primitive roots are successor ordinals.

Let $y_{1}, y_{2} \in \Sigma^{\text {Ord }}$ be two primitive strings such that $x=y_{1}^{\alpha_{1}}=y_{2}^{\alpha_{2}}$. The strings $y_{1}$ and $y_{2}$ are generalized cyclic by Proposition 4.3iii), which means

$$
y_{1}=\left(u^{\omega} v\right)^{\epsilon_{1}} u^{i_{1}} \text { and } y_{2}=\left(u^{\omega} v\right)^{\epsilon_{2}} u^{i_{2}}
$$

for some strings $u, v$, some integers $0 \leq i_{1}, i_{2}<\omega$ and some ordinals $\epsilon_{1}, \epsilon_{2}$ with $\epsilon_{1}=0$ if and only if $\epsilon_{2}=0$. The case $\epsilon_{1}=\epsilon_{2}=0$ is trivial since it implies $i_{1}=i_{2}$ and therefore $\alpha_{1}=\alpha_{2}$ by [10] (Cor. 7, p. 296), so we assume $\epsilon_{1}$ and $\epsilon_{2}$ different 
from 0 and by the previous claim we know that $\epsilon_{1}>1$ implies that $\epsilon_{1}$ is a prime component and likewise for $\epsilon_{2}$. Set $\lambda=\left|u^{\omega} v\right|$ and $\mu_{j}=\left|u^{i_{j}}\right|$ for $j=1,2$. Equality $y_{1}^{\alpha_{1}}=y_{2}^{\alpha_{2}}$ implies

$$
\left(\lambda \epsilon_{1}+\mu_{1}\right) \alpha_{1}=\left(\lambda \epsilon_{2}+\mu_{2}\right) \alpha_{2} .
$$

We have

$$
\left(\lambda \epsilon_{j}+\mu_{j}\right) \alpha_{j}=\left\{\begin{array}{cl}
\lambda \epsilon_{j} \alpha_{j}+\mu_{j} & \text { if } \alpha_{j} \text { is a successor ordinal } \\
\lambda \epsilon_{j} \alpha_{j} & \text { if } \alpha_{j} \text { is a limit ordinal. }
\end{array}\right.
$$

If $\alpha_{1}$ were a successor ordinal and $\alpha_{2}$ a limit ordinal then we would have $\lambda \epsilon_{1} \alpha_{1}+$ $\mu_{1}=\lambda \epsilon_{2} \alpha_{2}$. Because $\lambda>\mu_{1}$ holds we would get by Proposition 3.1, $\epsilon_{1} \alpha_{1}=\epsilon_{2} \alpha_{2}$ and $\mu_{1}=0$. Now condition $\mu_{1}=0$ implies $\epsilon_{1}=1$ by Claim 2 and thus $\alpha_{1}=\epsilon_{2} \alpha_{2}$. But this is a contradiction since $\alpha_{1}$ is a successor ordinal and $\epsilon_{2} \alpha_{2}$ is a limit ordinal. This establishes the third claim.

We first deal with the case where all exponents are successor ordinals and we show that the primitive root and its exponent are unique. Again we consider two roots like in (4). Equation (5), via (6), takes on the form $\lambda \epsilon_{1} \alpha_{1}+\mu_{1}=\lambda \epsilon_{2} \alpha_{2}+\mu_{2}$. Because of $\lambda>\mu_{1}, \mu_{2}$, Proposition 3.1 yields equalities $\epsilon_{1} \alpha_{1}=\epsilon_{2} \alpha_{2}$ and $\mu_{1}=\mu_{2}$. Then $\epsilon_{1}=1$ if and only if $\epsilon_{2}=1$ in which case we are done. We are left with $\epsilon_{1}=\omega^{\tau_{1}}$ and $\epsilon_{2}=\omega^{\tau_{2}}$ for some ordinals $\tau_{1}, \tau_{2}>0$. By equating the elements of lowest exponent in $\omega$ in Cantor's normal form in both handsides of equality $\epsilon_{1} \alpha_{1}=\epsilon_{2} \alpha_{2}$, we obtain $\tau_{1}=\tau_{2}$ which completes the proof in this case.

Now we assume all exponents associated with the primitive roots are limit ordinals. Consider the shortest string $y$ such that $x=y^{\alpha}$ for some (limit) ordinal $\alpha$. We verify that $y$ is strongly primitive. If this were not the case then by Definition 4.4 we could factor $y=z^{\omega} t$ with $z, t \in \Sigma^{\mathrm{Ord}}$ such that for some integer $0 \leq j<\omega$ and for some $\beta>1, z^{\omega} t z^{j}=w^{\beta}$, thus $|w|<|y|$ would hold. Since $\alpha$ is a limit ordinal we have $\left(z^{\omega} t\right)^{\alpha}=\left(z^{\omega} t z^{j}\right)^{\alpha}$ and thus $x=w^{\beta \alpha}$, contradicting the minimality of $|y|$. Furthermore, it is clear that all the strong conjugates of $y$ are strongly primitive roots of $x$.

Finally, let $z$ be another strongly primitive root of $x$. The strings $y$ and $z$ are generalized cyclic: $y=\left(u^{\omega} v\right)^{\beta} u^{i}$ and $z=\left(u^{\omega} v\right)^{\gamma} u^{j}$ for some strings $u, v$, for some integers $0 \leq i, j<\omega$ and for some ordinals $\beta, \gamma$ with $\beta=0$ if and only if $\gamma=0$. The only means of being strongly primitive is by assuming $\beta=\gamma=0$ and $i=j=1$ or $\beta=\gamma=1$ which concludes the proof.

\section{REFERENCES}

[1] A. Carpi and A. de Luca, Periodic-like words, periodicity and boxes. Acta Informatica 37 (2001) 597-618.

[2] Y. Césari and M. Vincent, Une caractérisation des mots périodiques. C. R. Acad. Sci. Paris A (1978) 1175-1177.

[3] C. Choffrut and S. Horváth, Transfinite equations in transfinite strings, 625-649. 
[4] J.P. Duval, Périodes et répétitions des mots du monoïde libre. Theoret. Comput. Sci. 9 (1979) 17-26.

[5] J.P. Duval, Mots de Lyndon et périodicité. RAIRO: Theoret. Informatics Appl. 14 (1980) 181-191.

[6] N.J. Fine and H.S. Wilf, Uniqueness theorems for periodic functions. Proc. Amer. Math. Soc. 3 (1965) 109-114.

[7] D. Giammarresi, S. Mantaci, F. Mignosi and A. Restivo, A periodicity theorem fro trees. Theoret. Comput. Sci. 1-2 (1998) 145-181.

[8] D. Klaua, Allgemeine Mengenlehre. Akademie Verlag (1969).

[9] J.G. Rosenstein, Linear ordering. Academic Press, New York (1982).

[10] W. Sierpiński, Cardinal and Ordinal Numbers. Warsaw: PWN (1958).

Received June, 2001. Revised March, 2002.

To access this journal online: www.edpsciences.org 